\title{
Educación a distancia y aprendizaje personalizado adaptativo
}

\section{Adaptive personalize distance education learning}

\author{
GUTIÉRREZ-TORNÉS, Agustín Francisco $\uparrow^{* 1}$, CANALES-CRUZ, Alejandro ${ }^{2}$ y ZAMBRANO- \\ DÁVILA, Juana Inés ${ }^{1}$
}

${ }^{1}$ Escuela Superior de Ciencias y Tecnologías de la Información, Universidad Autónoma de Guerrero. Lázaro Cárdenas 88 , Zona Sin Asignación Denombre de Col 21, Chilpancingo de los Bravo, Gro.

${ }^{2}$ Universidad Internacional de La Rioja, Campus México. Av. Universidad 472, Vértiz Narvarte, Benito Juárez, 03600 Ciudad de México, CDMX.

ID $1^{\mathrm{er}}$ Autor: Agustín Francisco, Gutiérrez-Tornés / ORC ID: 0000-0002-8634-9152, Researcher ID Thomson: X-22832018, CVU CONACYT ID: 251621

ID $1^{\text {er }}$ Coautor: Alejandro, Canales-Cruz

ID $2^{\text {do }}$ Coautor: Juana Inés, Zambrano-Dávila / ORC ID: 0000-0002-4526-4400, CVU CONACYT: 653891

DOI: $10.35429 / J B E .2019 .10 .3 .8 .14$

Recibido 27 de Octubre, 2019; Aceptado 20 de Diciembre, 2019

\section{Resumen}

El presente trabajo se sitúa en el ámbito de la educación virtual a distancia y más específicamente en una de las tecnologías cuyo uso ha aumentado exponencialmente. Son los sistemas de recomendación, que han comenzado a utilizarse en la educación como apoyo complementario en las plataformas diseñadas para brindar recursos de aprendizaje de forma más personalizada. La forma en que los estudiantes perciben, codifican, recuerdan, entienden y resuelven problemas es diferente entre cada uno. Esta tarea ha resultado ser un verdadero reto, tanto logístico como teórico, y la llegada de las nuevas tecnologías de la información se perfila como la opción más prometedora para proyectar y llevarla a cabo. Las investigaciones realizadas sobre el tema muestran un problema metodológico muy marcado. La forma en que se ha evaluado su efecto, especialmente en lo referente al proceso de aprendizaje, no ha sido desde ninguna óptica, rigurosa o exhaustiva. En general sólo se recurre a recabar opiniones de los estudiantes sobre cómo perciben que la utilización de dichos sistemas benefició este proceso. Parece importante conocer con mayor profundidad y precisión la forma en que el aprendizaje es potenciado con esta forma específica de tecnología.

Educación a distancia, Sistemas de recomendación, Personalización

\begin{abstract}
The present work is situated in the field of virtual distance education and more specifically in one of the technologies, whose use has increased exponentially. They are the recommendation systems, which are now used in education as a complementary support in the platforms designed to provide learning resources in a more personalized way. The way in which students perceive, codify, remember, understand and solve problems is different between each one. This task has proved a real challenge, both logistic and theoretical, and the arrival of new information technologies is emerging as the most promising option to project and carry it out. The investigations that have been carried out specifically in the subject show a very marked methodological problem. The way in which its effect has been evaluated, especially in relation to the learning process, has not been from any point of view, rigorous or exhaustive. In general, they only resort to obtaining opinions from students about how they perceive that the use of such systems benefited this process. It seems important to know in greater depth and precision the way in which learning is enhanced with this specific form of technology.
\end{abstract}

Distance education, Recommendation systems, Personalization

Citación: GUTIÉRREZ-TORNÉS, Agustín Francisco, CANALES-CRUZ, Alejandro y ZAMBRANO-DÁVILA, Juana Inés. Educación a distancia y aprendizaje personalizado adaptativo. Revista de Educación Básica. 2019. 3-10: 8-14.

\footnotetext{
*Correspondencia al Autor (Correo Electrónico: afgutierrezt@uagro.mx)

$\uparrow$ Investigador contribuyendo como primer autor.
} 


\section{Introducción}

Los seres humanos aprendemos de formas distintas, tales diferencias se hacen palpables en las preferencias individuales hacia distintos ambientes, métodos y medios con los que interactuamos en nuestro aprendizaje. Sumado a las diferentes preferencias en torno al aprendizaje, la sociedad de la información ha transformado las prácticas en diversos ámbitos, como el educativo, laboral y de consumo. En esas circunstancias, se ha marcado una gran división en cuanto a la percepción y habilidades propias de cada generación. Las exigencias de las Nuevas Generaciones de Estudiantes (NGE), como laNet ${ }^{1}$ y la Next ${ }^{2}$ presentan retos a los sistemas de educación masificados, que por lo general tienen un modelo de aprendizaje unitalla y de transferencia unidireccional (1).

En el Gráfico 1 se muestra una gráfica de la distribución por edades del alumnado de la Universidad Abierta y a Distancia de México (UnADM) que es uno de los principales centros representativos del auge de la enseñanza a distancia en México. De los 90,137 alumnos inscritos el $66.6 \%$ caen en el grupo de edad de la Generación Net lo que apoya la fundamentación de un cambio del Sistema Educativo a nivel Nacional.

Los recursos educativos disponibles en la Internet son muy numerosos y variados, por lo que podrían responder a las distintas necesidades, preferencias y estilos de aprendizaje de los estudiantes.

\footnotetext{
${ }^{1}$ La generación Net se considera a las personas nacidas de 1977 a 1997 (1), también generación Y o milenios. Los individuos pertenecientes a esta generación tienen las siguientes características (13): Habilidad para leer esquemas visuales, son comunicadores visuales intuitivos; capacidades viduales-espaciales derivadas de su experiencia con videojuegos, pudiendo integrar lo virtual y lo físico; descubrimiento inductivo, pues aprenden mejor descubriendo que siendo instruidos; despliegue de su atención, ya que pueden cambiar rápidamente de una tarea a otra, y pueden elegir no poner atención en cosas que no les interesan, y son capaces de responder rápidamente y esperar respuestas inmediatas.

${ }^{2}$ La generación Next pertenece a los nacidos de 1998 a la fecha (1)

ISSN-2523-2452

ECORFAN $^{\circledR}$ Todos los derechos reservados
}

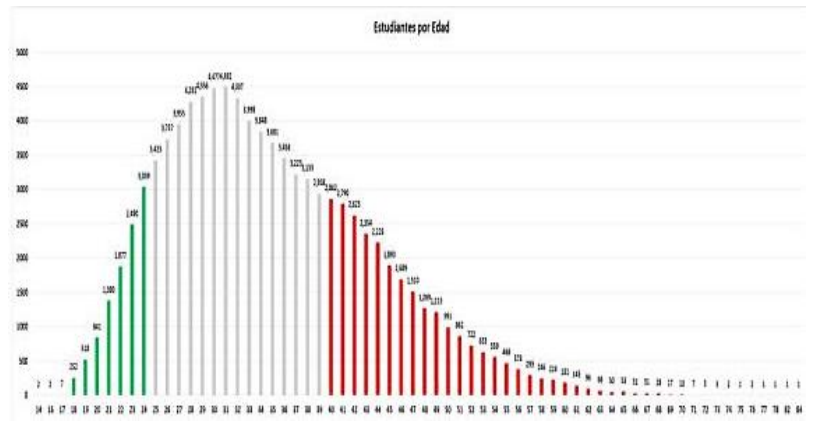

Figura 1 Distribución de la edad de la población estudiantil de la UnADM

Fuente: UnADM Estadísticas Abril, 2015

Desde el auge de Internet existen proyectos cuyo objetivo es el de obtener de forma personalizada los recursos de aprendizaje disponibles en la red y algunas de esas propuestas apuestan a los Estilos de Aprendizaje como eje para el éxito de los procesos de aprendizaje mediados por las Tecnologías de la Información y Comunicación (TIC). También, existen muchas tecnologías aplicadas a la educación que avanzan desde distintas perspectivas, que intentan otorgar a los estudiantes entornos personalizados que les doten de mayor autonomía en sus procesos de aprendizaje.

Debido a lo anterior, el presente trabajo busca integrar a las plataformas educativas basadas en TIC, mecanismos para la obtención de recursos de aprendizaje basados en el perfil de estilos de aprendizaje del usuario, de manera que se adapten a dicho estilo, en función del tiempo.

Este documento describe los resultados obtenidos producto de la investigación sobre el estado actual de los métodos y herramientas utilizados en el aprendizaje a distancia. Se desarrolló partiendo de tres distintos enfoques: según los diferentes Modelos de Estilos de Aprendizaje, a partir de los esfuerzos existentes para la elaboración de los Sistemas Hipermedia Adaptativos Educativos) y en los avances de los Sistemas de Recomendación.

Los resultados se presentan entonces en las partes que llevan su nombre. Finalmente se presentan unas conclusiones y el listado con las referencias bibliográficas. 


\section{Modelos de Estilos de Aprendizaje}

Los modelos y sistemas educativos apoyados en las TIC, especialmente la educación virtual a distancia, han limitado la personalización de la educación. Tales preferencias y comportamientos de las NGE, y con base en el contexto descrito en la anterior sección, es posible resaltar las siguientes problemáticas: diseño instruccional (el propio de la educación masificada y tradicional), de tal forma que queda limitada a la asesoría docente, compuesta por retroalimentaciones y evaluaciones.

La adaptación personalizada por cursos en línea, predominan en su mayoría los textuales, que son insuficientes o poco atractivos para las NGE. Los recursos textuales o hipertexto (según diversos estudios) no impactan o favorecen aprendizajes más efectivos (2) (3). Al ser, los recursos de aprendizaje poco atractivos en los entornos educativos cerrados, y ante la heterogeneidad de los mismos en la red, los estudiantes se encuentran en cantidades incalculables que dificulta su capacidad para elegir el más adecuado. Cada individuo posee su propio estilo de aprendizaje, según las competencias adquiridas (4). Estos sistemas suelen contar con una amplia oferta de actividades y recursos didácticos para los alumnos, pero aún con todo ello, muchas veces no son capaces de elegir la mejor opción para su estilo de aprendizaje.

Los Estilos de Aprendizaje se refieren a los distintos tipos de estrategias para aprender. Aunque éstas varían según lo que se desea aprender, el alumno desarrolla ciertas preferencias o tendencias globales las cuales definen un estilo de aprendizaje (visual, auditivo y kinestésico).

Los rasgos que definen a los estudiantes como perciben, interacciones y responden a sus ambientes de aprendizaje son: cognitivos, afectivos o fisiológicos. Los primeros tienen que ver con la forma en que los estudiantes estructuran el contenido, forman y utilizan conceptos, interpretan la información y resuelven problemas. Los afectivos son aquellos que se vinculan con la motivación y expectativas que influyen en el aprendizaje, y los fisiológicos se relacionan con el género y ritmo biológico.
Existen distintos modelos y teorías de aprendizaje que ofrecen marcos conceptuales que permiten entender los comportamientos diarios en el aula de los alumnos. Estos se clasifican de distinta manera todos tienen puntos en común los cuales permiten establecer estrategias para la enseñanza (5). En la Figura 2 se muestra los principales estilos de aprendizaje y su vinculación con las estrategias de enseñanza.

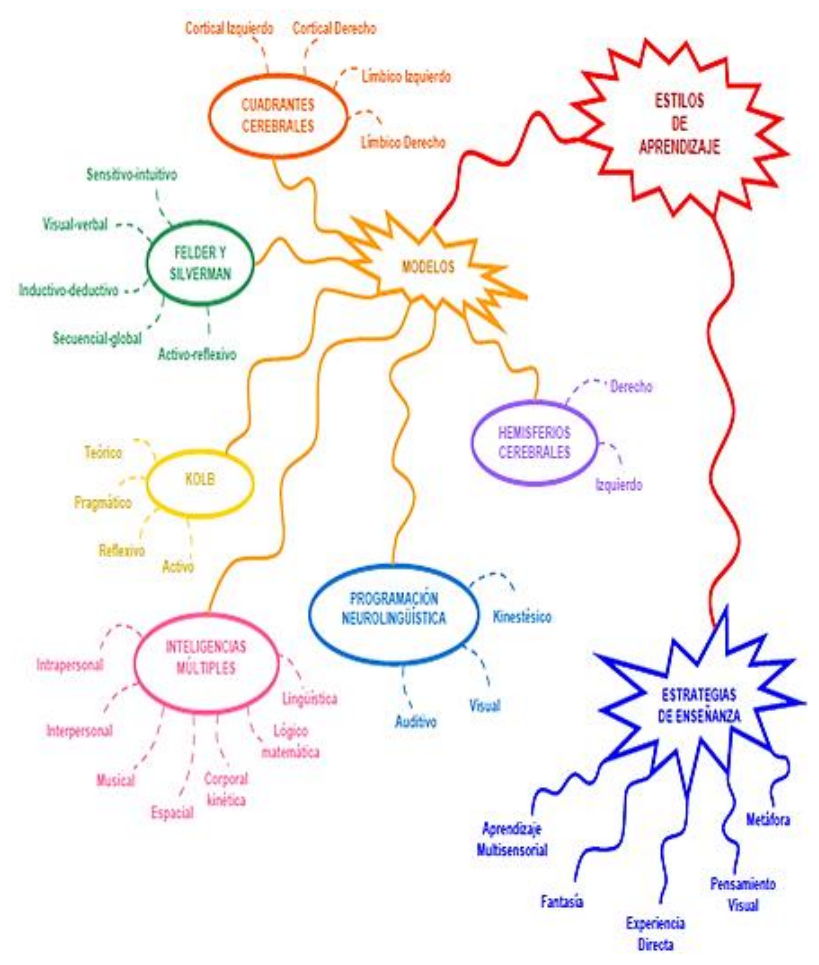

Figura 2 Principales Estilos de Aprendizaje Fuente: Gómez, L., Aduna, A., García, E., Cisneros, A., y Padilla, J. Manual de Estilos de aprendizaje (2004)

\section{Sistemas Adaptativos Inteligentes}

Los Sistemas Adaptativos Inteligentes para educación basados en la web se crean con la intención de solucionar el problema que se acaba de enumerar: la elección de la mejor opción a la que se adapte un estilo de aprendizaje determino, automatizando la adaptación del sistema a las características y necesidades de los usuarios. Utilizan las técnicas de los sistemas adaptativos nos ayuda para construir un modelo de alumno el cual le permite adaptar el sistema a sus necesidades de aprendizaje, aplicando técnicas de inteligencia artificial (IA) para realizar tareas tales como el adiestramiento, la monitorización de los alumnos y el diagnóstico de sus limitaciones (5). 
Para abordar este problema, se han utilizado con éxito técnicas de descubrimiento de conocimiento o minería de datos que asisten al profesor en la validación de los recursos puestos a disposición en el curso. El desarrollo de un Sistema Adaptativo Inteligente es una actividad laboriosa, tanto más compleja cuando mayor es el número de posibilidades de adaptación que se desea ofrecer. Cabe recalcar que también se hace necesaria realizar una evaluación posterior basada en que califique los resultados obtenidos tras la utilización del sistema.

\begin{tabular}{|c|c|}
\hline $\begin{array}{l}\text { Sistema, autor, } \\
\text { año }\end{array}$ & $\begin{array}{c}\text { Modelo de estilos de aprendizaje } \\
\text { y adaptación }\end{array}$ \\
\hline $\begin{array}{l}\text { 1. CS383 } \\
\text { Carver et al } \\
1994\end{array}$ & $\begin{array}{l}\text { Modelo de estilos de aprendizaje: } \\
\text { Construcción de modelo } \\
\text { metodológico y prototipo de } \\
\text { validación para el diseño de } \\
\text { SHAA basados en Web, en } \\
\text { función de } \\
\text { aprendizaje y estilos cognitivo } \\
\text { Felder-Silverman } \\
\text { (sensorial/intuitivo, visual/verbal y } \\
\text { secuencial/global). } \\
\text { Adaptación: Los resultados } \\
\text { muestran una mejora en el grupo } \\
\text { experimental (diseñado para la } \\
\text { adaptación), pero deja abierta la } \\
\text { línea para validar en otros } \\
\text { contextos. }\end{array}$ \\
\hline $\begin{array}{l}\text { 2. ARTHUR } \\
\text { Gilbert y Han } \\
1999\end{array}$ & 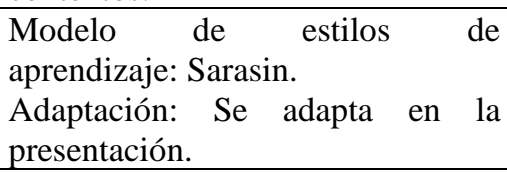 \\
\hline $\begin{array}{l}\text { 3. MANIC } \\
\text { (Multimedia } \\
\text { Asynchronous } \\
\text { Networked } \\
\text { Individualized } \\
\text { Courseware) } \\
\text { Sternberg et al } \\
\text { 2000 }\end{array}$ & $\begin{array}{l}\text { Modelo de estilos de } \\
\text { aprendizaje: Basado en distintos } \\
\text { aspectos de varios Modelos de } \\
\text { Estilos de Aprendizaje. } \\
\text { Adaptación: Se basa en el texto } \\
\text { expandible. Adapta el tipo de } \\
\text { objeto multimedia, la instrucción y } \\
\text { el lugar dentro de la estructura. }\end{array}$ \\
\hline $\begin{array}{l}\text { 4. IDEAL } \\
\text { (Intelligent } \\
\text { Distributed } \\
\text { Environment } \\
\text { for Active } \\
\text { Learning) } \\
\text { Shang et al } \\
2001\end{array}$ & 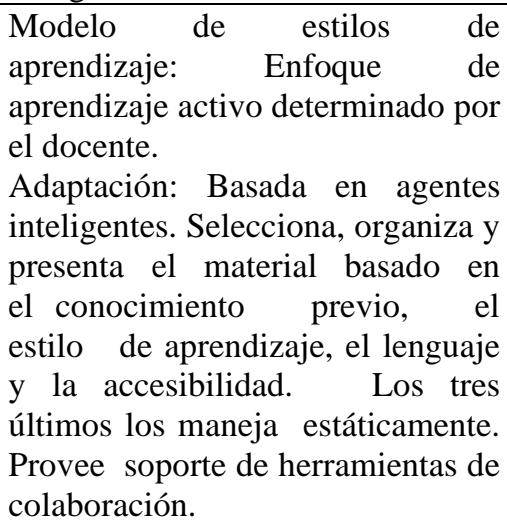 \\
\hline
\end{tabular}

\begin{tabular}{|c|c|}
\hline $\begin{array}{l}\text { 5. MASPLANG } \\
\text { Peña } \\
2002\end{array}$ & $\begin{array}{l}\text { Modelo de estilos de } \\
\text { aprendizaje: Felder-Silverman. } \\
\text { Adaptación: Sistema multi-agente } \\
\text { para enriquecer el USD (Sistema } \\
\text { tutor inteligente). La adaptación se } \\
\text { da en la combinación de formatos } \\
\text { multimedia, estrategias de } \\
\text { enseñanza y herramientas de } \\
\text { navegación. }\end{array}$ \\
\hline $\begin{array}{l}\text { 6. LSAS } \\
\text { (Learning Style } \\
\text { Adaptive } \\
\text { System) } \\
\text { Bajraktarevic } \\
\text { et al } \\
2003\end{array}$ & $\begin{array}{l}\text { Modelo de estilos de aprendizaje: } \\
\text { La dimensión secuencial/global de } \\
\text { Felder-Silverman. } \\
\text { Adaptación: Se provee con dos } \\
\text { plantillas; una basada en texto y } \\
\text { enlaces secuenciales; y la segunda } \\
\text { consta de organizadores } \\
\text { avanzados, retroalimentación y } \\
\text { máxima instrucción. }\end{array}$ \\
\hline $\begin{array}{l}\text { 7. iWeaver } \\
\text { Wolf } \\
2003\end{array}$ & $\begin{array}{l}\text { Modelo de estilos de aprendizaje: } \\
\text { Building Excellence } \\
\text { Adaptación: Busca mantery. } \\
\text { equilibrio en la carga cognitiva, } \\
\text { ordenar y ocultar las opciones } \\
\text { de navegación y y los } \\
\text { contenidos. } \\
\end{array}$ \\
\hline $\begin{array}{l}\text { 8. INSPIRE } \\
\text { (Intelligent } \\
\text { System for } \\
\text { Personalized } \\
\text { Instruction in } \\
\text { a Remote } \\
\text { Environment) } \\
\text { Papanikolau. } \\
2003\end{array}$ & $\begin{array}{l}\text { Modelo de estilos de } \\
\text { aprendizaje: Honey y Mumford } \\
\text { (activo, teórico, pragmático y } \\
\text { reflexivo). } \\
\text { Adaptación: En la secuencia de } \\
\text { contenidos, en las opciones de } \\
\text { navegación y en la presentación de } \\
\text { los contenidos. Permite a los } \\
\text { estudiantes seleccionar los } \\
\text { objetivos de aprendizaje y genera } \\
\text { lecciones en base a sus } \\
\text { conocimientos previos, progreso } \\
\text { y estilo de aprendizaje. }\end{array}$ \\
\hline $\begin{array}{l}\text { 9. MOT } \\
\text { (My Online } \\
\text { Teacher) Stash } \\
\quad \text { et al. } \\
2006\end{array}$ & $\begin{array}{l}\text { Modelo de estilos de } \\
\text { aprendizaje: Activo, pragmático, } \\
\text { reflexivo y teoría de Kolb. } \\
\text { Adaptación: Modelo de tres capas: } \\
\text { la autoría de adopción (capa } \\
\text { jerárquica conceptual, capa de } \\
\text { lecciones y capa de adaptación de } \\
\text { estudiante y presentación. }\end{array}$ \\
\hline $\begin{array}{l}\text { 10. AHA! } \\
\text { (Adaptive } \\
\quad \text { Hypermedia } \\
\quad \text { for All) } \\
\text { De Bra et al. } \\
1998\end{array}$ & $\begin{array}{l}\text { Modelo de estilos de aprendizaje: } \\
\text { Permite elegir el modelo de estilos } \\
\text { de aprendizaje por aplicar (utiliza } \\
\text { estrategias educativas } \\
\text { predefinidas según los estilos de } \\
\text { aprendizaje: activo / reflexivo, } \\
\text { verbales/visuales, } \\
\text { holísticos/analíticos y dependientes } \\
\text { /independientes de campo) } \\
\text { determinado por el docente. } \\
\text { Adaptación: } \\
\text { herramienta de Desarrolla } \\
\text { autor y lenguaje de adaptación } \\
\text { de estilos de aprendizaje llamado } \\
\text { LAG-XLS. }\end{array}$ \\
\hline
\end{tabular}
distancia y aprendizaje personalizado adaptativo. Revista de Educación Básica. 2019 


\begin{tabular}{|c|c|}
\hline $\begin{array}{l}\text { 11. TANGOW } \\
\text { (Task-based } \\
\text { Adaptive } \\
\text { learNer Guidan- } \\
\text { ce On the } \\
\text { Web) Carro et } \\
\text { al.- Paredes. } \\
\text { 1999-2008 }\end{array}$ & $\begin{array}{l}\text { Modelo de estilos de aprendizaje: } \\
\text { Felder y } \\
\text { (secuencial/global } \\
\text { sensorial/intuitivo). } \\
\text { Adaptación: Adapta tanto las } \\
\text { interacciones como el seguimiento } \\
\text { del curso. El diseñador elige las } \\
\text { tareas, tipo de reglas, así como su } \\
\begin{array}{l}\text { secuencia, requisitos previos y } \\
\text { obligatoriedad. }\end{array}\end{array}$ \\
\hline $\begin{array}{l}\text { 12. CoMoLE } \\
\text { (Context-based } \\
\text { adaptive } \\
\text { Mobile } \\
\text { Learning } \\
\text { Environment) } \\
\text { Martín. 2008 } \\
\end{array}$ & \begin{tabular}{llr} 
Modelo de & \multicolumn{1}{c}{ estilos } & de \\
aprendizaje: & Felder-Silverman \\
(sensorial/intuitivo & y \\
visual/verbal). & & \\
$\begin{array}{l}\text { Adaptación: } \\
\text { recomendación con monismo }\end{array}$ & de \\
adaptación basado en reglas. & \\
\end{tabular} \\
\hline $\begin{array}{l}\text { 13. G-OALS } \\
\text { (Online } \\
\text { Analyzer of } \\
\text { Learning Styles) } \\
\text { Ferrer et al. } \\
2013\end{array}$ & $\begin{array}{l}\text { Modelo de estilos de } \\
\text { aprendizaje: Felder y Silverman } \\
\text { (visual/verbal, secuencial/global y } \\
\text { sensorial/intuitivo). } \\
\text { Adaptación: El diseñador elige los } \\
\text { contenidos y el sistema los } \\
\text { presenta de acuerdo a los estilos de } \\
\text { aprendizaje detectados. }\end{array}$ \\
\hline
\end{tabular}

Tabla 1 Sistemas Hipermedia Adaptativos Educativos identificados

Fuente: elaboración propia

En la Figura 3 se muestra un mapa de la evolución de los Sistemas Hipermedias Adaptativos Educativos.

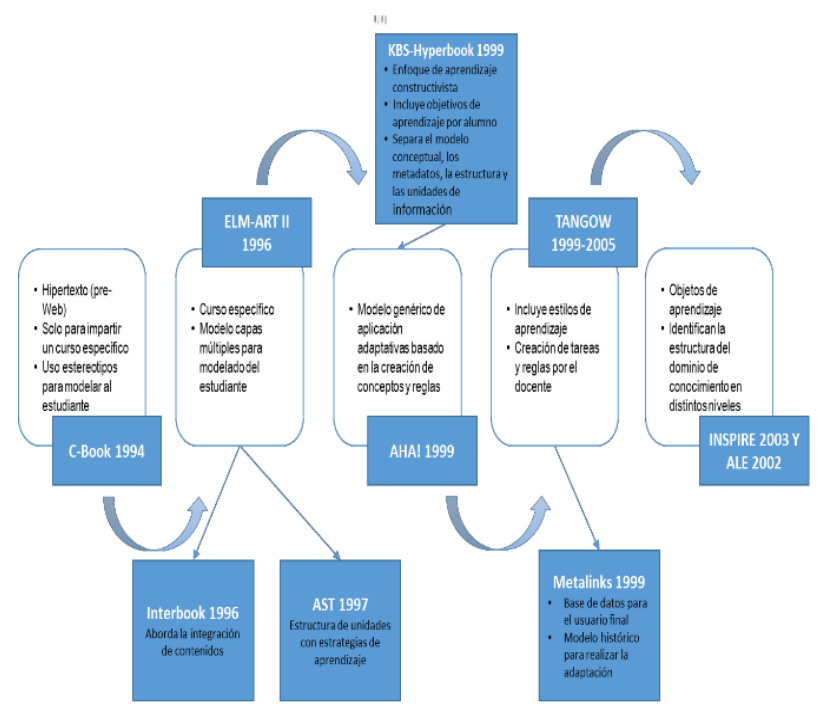

Figura 3 Evolución de los Sistemas Hipermedia Adaptativos Educativos

Fuente: Berlanga (2006)

\section{Sistema de Recomendación}

Los Sistemas de Recomendación surgen por la década de los noventa con el fin de proporcionar a los usuarios resultados de búsquedas de información cercanos a sus requerimientos.
Constituyen una forma de personalización desde el punto de vista computacional, ya que es un sistema capaz de tratar cada usuario de manera individual, adecuando un producto o servicio para atender las necesidades de cada uno de manera diferente. Es una de las formas más promisorias para mejorar la calidad de las búsquedas. Son capaces de identificar preferencias y sugerir ítems relevantes para cada usuario; para ello se necesita de perfiles que almacenen la información y las preferencias de cada uno.

En el contexto de la Educación a Distancia, existen diferentes tipos de Sistemas de Recomendación Educativos (SRE), los cuales pueden ser usados para diferentes enfoques y con diferentes contextos. Se enumeran y describen brevemente los principales tipos:

- Basados en el Contenido de los Objetos de Aprendizaje que son una tecnología instruccional basada en el paradigma orientado a objetos, el cual se refiere a crear componentes o módulos que puedan ser reutilizables por otros programas educativos. Se corresponden con el concepto de actividad de aprendizaje y se conforman por una información digital donde se refleja su contenido (datos) así como de información descriptiva, administrativa y estructural que facilita su utilización (metadatos). El estilo de aprendizaje puede considerarse un metadato (6) (7) (8).

- Colaborativos. Las recomendaciones se hacen basándose en el grado de similitud entre usuarios. Esto se fundamenta en el hecho de que los Objetos de Aprendizaje que le gustan a un usuario, le pueden interesar a otros. Esta clase de sistemas construyen la recomendación como una agregación estadística/probabilística de las preferencias de otros usuarios que ofrezca recomendaciones de calidad, utilizando un buen algoritmo de filtrado colaborativo (9) (7) (10) (11). 
- Basados en el Conocimiento. Un sistema de recomendación basado en conocimiento, hace recomendaciones según el historial de navegación del usuario, el cual está almacenado con el fin de obtener las preferencias e intereses y con ello obtener la información necesaria para generar recomendaciones. Estos son llamados Sistemas de Preferencias Implícitas ya que deducen las preferencias a partir del comportamiento del usuario y de su historial. Esto permite que en la mayoría de los casos no sea necesario pedir al usuario demasiada información sobre sus preferencias para que pueda ser recomendado (7).

- Híbridos. El enfoque híbrido, busca la relación entre varios enfoques o técnicas de recomendación con el objetivo de complementar sus principales características y realizar la mejor recomendación (8). A continuación, se mencionan algunos métodos de combinación o integración como:

- Método ponderado: Donde se combinan las puntuaciones o votos para producir una única recomendación.

- Método de Conmutación: El sistema conmuta entre las técnicas de recomendación en función de la situación actual.

- Método Mixto: Se presentan las recomendaciones de diferentes sistemas de recomendación al mismo tiempo.

- Método de combinación de características de diferentes fuentes de datos se entregan como entradas a un único algoritmo de recomendación.

- Método de cascada: Cada una de las recomendaciones refina las recomendaciones dadas por los otros.

- Función de aumento: Una característica de salida de una técnica, se usa como una característica de entrada a otra.

○ Meta-nivel: El modelo aprendido por un sistema de recomendación se utiliza como entrada a otro (7).

\section{Conclusiones}

Las NGE han vivido en una sociedad en donde la tecnología ha sido parte fundamental de su desarrollo como individuos, como miembros de una sociedad globalizada e interconectada por medios electrónicos; se han encontrado con un sistema educativo que se desarrolla en una inercia que vuelve muy difícil el ajustarse a las necesidades actuales de la sociedad, y mucho más a los estándares planteados por los países industrializados.

Un ejemplo de lo anterior, se observa en los Estándares Nacionales (EEUU) de Tecnologías de Información y Comunicación (TIC) para estudiantes, definidos por ISTE 7, que establecen seis puntos que los estudiantes deben de cubrir para poder aprender y hacer, en un mundo cada día más digital. Los seis puntos son:

1. Creatividad e innovación.

2. Comunicación y colaboración.

3. Investigación y manejo de información.

4. Pensamiento crítico, solución de problemas $\mathrm{y}$ toma de decisiones.

\section{Ciudadanía digital.}

6. Funcionamiento y conceptos de las TIC.

Los resultados en la educación, como se menciona en la contextualización, no han dado resultados positivos que evidencien que los estudiantes se adapten del todo a las viejas estrategias de promoción del aprendizaje.

Para las modalidades educativas basadas en TIC, los recursos de aprendizaje son un elemento fundamental que, cohesionados con la definición de objetivos, estrategias y actividades de aprendizaje de las unidades de formación, de los programas educativos, generan el insumo de información básico; dichos programas educativos, en la mayoría de los casos, no toman en cuenta el perfil de estilo de aprendizaje de los estudiantes. 
Aunque se pueden complementar los conocimientos adquiridos con recursos disponibles en la Red de Redes, debido a la infoxicación a la que estamos expuestos, es muy difícil encontrar recursos adecuados a nuestras preferencias sensoriales y cognitivas, por tanto, se hace necesaria la personalización adaptativa de los recursos de aprendizaje, y mayormente en los entornos educativos mediados por TIC. Como respuesta a lo anterior, es necesario replantear estrategias y modelos de aprendizaje, lo cual representa uno de los principales retos para la educación en las modalidades mixtas, en línea y/o a distancia, ya que las NGE ingresan cada vez más en dichas modalidades. Por lo anterior, es necesario mantener su atención para lograr un aprendizaje significativo, y al ser la tecnología uno de sus principales medios de interacción con el mundo, se debe aprovechar su impacto en las modalidades de aprendizaje mediadas por la tecnología (mixta y en línea), siendo los recursos de aprendizaje un elemento base.

\section{Agradecimientos}

Los autores de este trabajo desean expresar su reconocimiento a la Universidad Autónoma de Guerrero, en especial a la dirección de la Escuela Superior de Ciencias y Tecnologías de la Información, y a la Universidad Internacional de La Rioja, Campus México, el apoyo brindado para su realización.

\section{Referencias}

(1) Tapscott, D. (2008). Grown up digital. Boston: McGraw-Hill Education.

(2) Chen, S. Y. H., \& Ford, N. J. (1997). Towards adaptive information systems: individual differences and hypermedia. Information Research, 3(2), 3-2.

(3) Karagiannidis, C., \& Sampson, D. (2004, August). Adaptation rules relating learning styles research and learning objects meta-data. In Workshop on Individual Differences in Adaptive Hypermedia. 3rd International Conference on Adaptive Hypermedia and Adaptive Web-based Systems (AH2004), Eindhoven, Netherlands.

(4) Alonso, C., Gallego, D., y Honey, P. (1995). Los estilos de aprendizaje. Procedimientos.
(5) Angulo, M. P. (2005). Educación a distancia en el siglo XXI. Apertura

(6) Cazella, S. C., Reategui, E. B., \& Nunes, M. A. (2010). A Ciência da Opinião: Estado da arte em Sistemas de Recomendação. JAI: Jornada de Atualização em Informática da SBC. Rio de Janeiro, RJ: PUC Rio, 161-216.

(7) Wang, T. I., Tsai, K. H., Lee, M. C., \& Chiu, T. K. (2007). Personalized Learning Objects Recommendation based on the Semantic Aware Discovery and the Learner Preference Pattern. Educational Technology \& Society, 10(3), 84-105.

(8) Mizhquero, K. (2009). Análisis, Diseño e Implementación de un Sistema Adaptivo de Recomendación de Información Basado en Mashups. Revista Tecnológica ESPOL.

(9) Burke, R. (2002). Hybrid Recommender Systems: Survey and Experiments. User Modeling and User-Adapted Interaction

(10) Álvarez Basilio, E. (2018). Modelo Ontológico para bases de datos utilizadas en un generador de Sistemas de Recomendación. Tesis de Grado, UACyTIUAGro.

(11) Tornés, A. F. G., Cruz, A. C., UnADM, D. D., y Dávila, M. J. I. Z. Sobre el estado actual de los métodos y herramientas utilizados en el aprendizaje a distancia.

(12) Oblinger, D., Oblinger, J. L., \& Lippincott, J. K. (2005). Educating the net generation. Boulder, Colo.: EDUCAUSE 